\title{
Evaluation of Analgesic Effect of Transversus Abdominis Plane Block after Emergency Cesarean Section
}

\author{
M.O.Mashal, N.E.I.Ashmawy, A.A.Shdeed and M.A.El-gazzar \\ Gynecology and Obstetrics, Dept., Faculty of Medicine, Benha Univ., Benha, Egypt \\ E-mail: mohamed.meshel@yahoo.com
}

\begin{abstract}
Background: Adequate postoperative analgesia is crucial after a caesarean section (CS) to facilitate early ambulation and newborn care (breast feeding, maternal-fetal bonding), as well as to reduce morbidity. As part of a multimodal analgesic strategy, the transversus abdominis plane (TAP) block is used in CS postoperative analgesia. The goal of our study is to see how effective TAP block is as an analgesic in the first 48 hours following emergency CS by Pfannenstiel incision. $n$ Methodologies: A total of 100 pregnant women receiving emergency CS were enrolled in this prospective double-blind trial. They were divided into two groups at random. Group A: Bupivacaine was used to inhibit TAP in 50 women. Group B (control group): 50 women were given TAP block with 0.9 percent saline. Results: At rest and during movement, VAS in group B was substantially lower than in group A, although there was no significant difference between the two groups at baseline. When compared to group A, the time to the first morphine request was greatly delayed, and the overall dosage of morphine was greatly reduced. At 12 hours and 1 day, group B had much less nausea and sedation than group A, but at 0 and 2 days, there was no significant difference between the two groups. Final Thoughts: The use of an ultrasound-guided TAP block following emergency CS lowers the need for analgesics in the first 48 hours following surgery. It reduces pain intensity, lengthens the time to initial rescue analgesia, and lowers overall morphine, sedation, and nausea scores.
\end{abstract}

Keywords: Transversus Abdominis Plane Block, Emergency, Cesarean Section.

\section{Introduction}

Post-operative discomfort that is not well controlled may lead to complications and a long recovery [1]. Uncontrolled pain may lead to chronic discomfort and a reduction in overall life quality [2]. The patient's recuperation and capacity to resume regular functional activities is hindered as a consequence [3].

Patients who are expected to care for their infant right after surgery must recuperate quickly. According to studies undertaken in high-income nations, inadequately managed pain after a caesarean section (CS) has been associated to an increased prevalence of chronic pain [4]. It also causes post-traumatic stress disorder [5].

A range of variables contribute to the failure to provide appropriate postoperative analgesia. Inadequate knowledge, fear of analgesic medication side effects, inadequate pain assessment, and a lack of personnel are just a few of the causes behind this. The goal of postoperative pain management is to reduce discomfort while limiting side effects [6].

The effectiveness of a transversus abdominis plane (TAP) block in managing postoperative pain for procedures involving a midline abdominal wall incision was shown. As part of a multimodal analgesic treatment for CS, TAP block has a lot of promise [7].

Our goal is to see how important TAP block is in the first 48 hours following a Pfannenstiel incision for emergency CS.

\section{Patients and methods}

In this prospective double-blinded, randomised, controlled experiment at Banha University Hospital in Egypt, 100 pregnant women aged over 18 years old, emergency CS, full term pregnancy, haemoglobin level $10 \mathrm{gm} / \mathrm{dl}$ (or higher) were enrolled.

After receiving clearance from the institutional ethics committee, each participant gave informed written permission after being told of the study's objectives and methods.

Exclusion criteria included a history of relevant medication allergies and medical treatments that were thought to result in opioid tolerance.

Patients were separated into two groups and assigned at random. Group A consisted of 50 women who had their TAP blocked with bupivacaine. Group $\mathrm{B}$ : In the control group, 50 women were given TAP block using 0.9 percent saline.

\subsection{All cases were subjected:}

1. Careful and detailed history: Personal history, obstetric history (first day of last menstrual period and estimated gestational age), past history of diseases and surgical history (previous uterine scar and previous laparotomy).

\section{Examination of the patients:}

General examination (maternal body Wight, height, presence of abnormal color e.g. pallor or jaundice, vital data (blood pressure, pulse, temperature), cardiac and chest examination and petechiae or ecchymosis of the skin to exclude coagulopathy) and Abdominal examination (size of the uterus, scar of previous laparotomy), vaginal examination after operation: to confirm uterine contraction and to exclude postpartum hemorrhage postoperative.

3. Investigation: $\mathrm{ABO}$ grouping, $\mathrm{Rh}$ typing, $\mathrm{CBC}$ for Hemoglobin, and Hematocrit values.

4. Indication of C.S: Fetal distress, obstructed labor/failure to progress, breech presentation, 
antepartum hemorrhage, transverse lie, cephalopelvic disproportion, cord prolapse, twin and first breech presentation and previous two cesarean section with contracting uterus.

Procedures: The 100 pregnant women that underwent emergency CS under spinal anesthesia using $2.2 \mathrm{ml}$ of $0.5 \%$ hyperbaric bupivacaine along with $20 \mathrm{mg}(0.4 \mathrm{ml})$ of fentanyl. Spinal anesthesia and TAP block was done by same anesthetist. The women were divided to 2 groups: Group A (control group): 50 pregnant women underwent TAP block using $20 \mathrm{ml}$ of $0.9 \%$ saline. Group B (study group): 50 pregnant women underwent TAP block with $20 \mathrm{~mL} 0.25 \%$ bupivacaine each side; dose 100mg. TAP block was performed at the end of surgery.

Requirements are US machine with a high frequency probe $(5-10 \mathrm{MHz})$, US probe cover, antiseptic for skin disinfection, sterile US gel, spinal needle Quincke type point (22GA, $0.7 \times 90 \mathrm{~mm}$, sterile, disposable, made in Spain) and $20 \mathrm{ml}$ syringe and injection tubing.

After skin and transducer preparation, the transducer was positioned above the iliac crest in an axial (transverse) plane. The internal oblique, transversus, and intervening TAPs can all be easily identified at this point and can be followed laterally to the region above the iliac crest where the block was performed.

In plane with the transducer, in an anteriorposterior direction a spinal needle Quincke type point (22GA, $0.7 \times 90 \mathrm{~mm})$, sterile, disposable was inserted.

The visualization of a hypoechoic fluid immediately deep to the hyperechoic facial plane below the internal oblique is an indication of correct needle tip insertion and deposition of LA.

Instead of the correct plane, if the needle tip was intramuscular, a pattern of fluid spread consistent with intramuscular injection was seen instead. A total of 20 ML $0.25 \%$ bupivacaine each side; dose $100 \mathrm{mg}$ was injected in the first group (group A), and $20 \mathrm{ml}$ of 0.9 $\%$ saline in the second group (group B) into this plane on each side.

During LA injection, the abdomen was scanned cephalic and caudal to determine the extent of longitudinal spread of the injectate, medial to lateral scanning was determined the extent of horizontal spread of the injectate.

Using spinal needle Quincke type point (22GA, $0.7 \times 90 \mathrm{~mm}$, sterile, disposable, made in Spain), this needle was attached to syringe filled with the study solution under US guide. After completion of surgery and TAP block the routine post-operative analgesia was given. This consisted of paracetamol $1 \mathrm{gm} \mathrm{IV}$ every 6 hours and diclofenac 50mg per oral every 8 hours according to Banha university hospital protocol. Intravenous morphine was commenced to the patient upon demand with maximum dose of $8 \mathrm{mg}$ every time which can be repeated after a period not less than 6 hours. Total dose of morphine was calculated in 48 hours in mgs. The presence and severity of nausea, and sedation will be assessed systemically by investigator who will be blinded to group allocation. The assessment will be performed in the post anesthesia care unit (PACU) and in the department at 12,24 and $48 \mathrm{~h}$ after the TAP blockade. All patients will be asked to give score for their pain at rest and on movement (knee flexion) and the degree of nausea at each time point. Pain severity will be measured using both a visual analog scale (VAS) $0 \mathrm{~cm}$ unmarked line in which $0 \mathrm{~cm}=$ no pain and $10 \mathrm{~cm}$ worst pain imaginable) and categorical pain scoring system (none $=0$; mild-1; moderate $=2$; sever $=3$ ). Nausea will be measured using categorical scoring system (none $=0$; mild $=1$; moderate $=2$; sever $=3$ ). Sedation score will be assigned by the investigator using a sedation scale (awake and alert $=0$; quietly awake $=1$; asleep $=$ but easily roused $=2$; deep sleep $=3$ ). [7]

Primary outcome is cumulative morphine consumption after 48 hours' post-operative.

Secondary outcomes are cumulative morphine consumption after $12,24,48$ hours' post-operative. Continuous morphine consumption over time and time to first patient-administered morphine bolus. Side effects (nausea, vomiting, pruritus and sedation). Pain at rest, mobilization and the use of antiemetic medication

\subsection{Sample size justification}

Sample size was100 cases, calculated based on the following criteria: $95 \%$ confidence level, $80 \%$ power of the study and group to group ratio 1:1.

\subsection{Statistical analysis}

SPSS v25 (IBM Inc., Chicago, IL, USA) was used for statistical analysis. The mean and standard deviation (SD) of numerical variables were calculated and compared between the two groups using the student's t-test. Categorical variables were presented as number (\%) and were analysed using the Chisquare test or Fisher's exact test as needed. It was considered significant if the two-tailed $\mathrm{P}$ value was less than 0.05 .

\section{Results}

In this study, 118 women were assessed for eligibility, 11 women did not meet the criteria and 7 women refused to participate in the study. One hundred women were randomly allocated into two groups (50 women in each one), followed-up and analyzed statistically Fig. (1).

The age, weight, duration of $\mathrm{CS}$, primigravida and multigravida in both groups were insignificantly different Table (1)

VAS at rest was significantly decreased in group B compared to group A at 12, 24 and 48 hours ( $P$ $<0.001)$ but was insignificantly different between both groups at baseline (Table 2) 
VAS at movement was significantly decreased in group B compared to group A at 12, 24 and 48 hours $(\mathrm{P}<0.001)$ but was insignificantly different between both groups at baseline Table (2)

The total dose of morphine ranged between 20 and $40 \mathrm{mg}$ with a mean value of $28.10 \pm 5.43 \mathrm{mg}$ in group $\mathrm{A}$ and ranged between 10 and $15 \mathrm{mg}$ with a mean value of $12.80 \pm 1.84 \mathrm{mg}$ in group $\mathrm{B}$. The total dose of morphine was significantly decreased in group B compared to group A (P<0.001) Table (3)

The time to the first morphine request ranged between 1.5 and 3.5 hours with a mean value of 2.19 \pm 0.55 hours in group A and ranged between 3.75 and 7.5 hours with a mean value of $5.70 \pm 1.34$ hours in group $\mathrm{B}$. The time to the first morphine request was significantly delayed in group B compared to group A (P <0.001) Table (3)

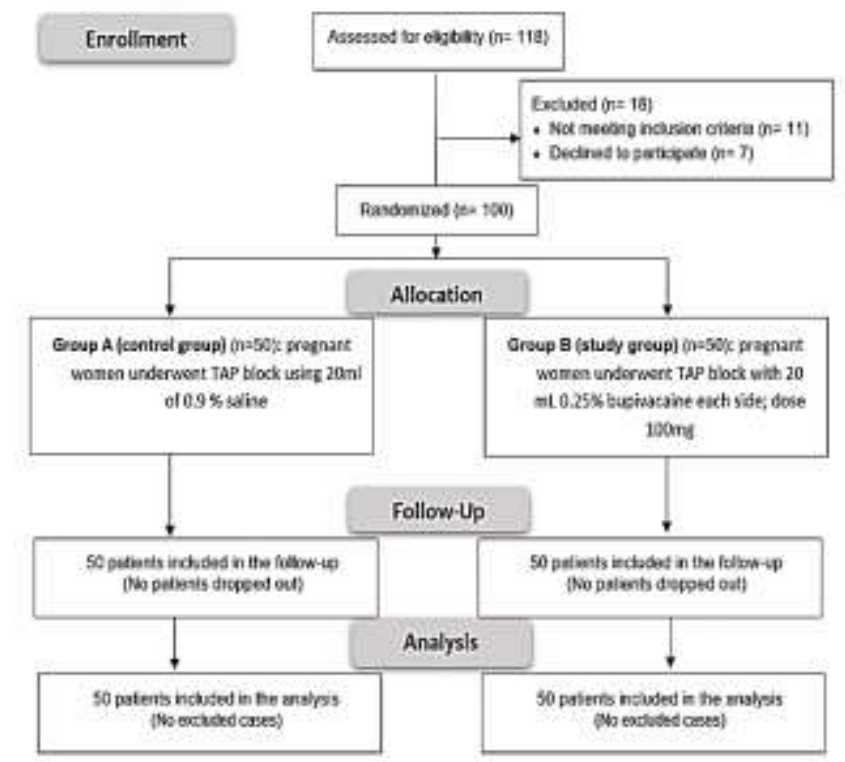

Fig. (1) CONSORT flow diagram.

Table (1) Patients' characteristics in both studied groups.

\begin{tabular}{llccc}
\hline & Group A & $\begin{array}{c}\text { Group B } \\
(\mathbf{n}=\mathbf{5 0})\end{array}$ & P value \\
\hline Age (years) & Mean + SD & $27.14 \pm 4.87$ & $27.94 \pm 5.05$ & 0.422 \\
& Range & $20-35$ & $20-35$ & \\
Weight (kg) & Mean \pm SD & $84.98 \pm 9.11$ & $83.84 \pm 8.88$ & 0.528 \\
Duration of & Meange & $70-99$ & $70-99$ & \\
$\begin{array}{l}\text { cesarean section } \\
\text { (min) }\end{array}$ & Range & $54.70 \pm 14.79$ & $56.06 \pm 14.45$ & 0.643 \\
Gravidity & Primigravida & $30-80$ & $31-80$ & \\
& Multigravida & $27(54 \%)$ & $33(66 \%)$ & 0.221 \\
\hline
\end{tabular}

Table (2) Visual analogue scale (VAS) at rest and at movement in both studied groups.

\begin{tabular}{|c|c|c|c|c|c|}
\hline & & Baseline & $12 \mathrm{~h}$ & $24 \mathrm{~h}$ & $48 \mathrm{~h}$ \\
\hline \multicolumn{6}{|l|}{ At rest } \\
\hline Group A & Mean & 1.3 & 5.2 & 3.7 & 2.5 \\
\hline$(\mathbf{n}=\mathbf{5 0})$ & \pm SD & 0.9 & 0.8 & 0.9 & 0.6 \\
\hline Group B & Mean & 1.0 & 2.7 & 1.3 & 1.2 \\
\hline \multirow{2}{*}{$(\mathbf{n}=\mathbf{5 0})$} & $\pm \mathrm{SD}$ & 0.7 & 1.3 & 0.5 & 0.4 \\
\hline & & 0.093 & $<0.001 *$ & $<0.001 *$ & $<0.001 *$ \\
\hline \multicolumn{6}{|c|}{ At movement } \\
\hline Group A & Mean & 3.2 & 6.8 & 5.6 & 4.5 \\
\hline$(\mathbf{n}=\mathbf{5 0})$ & $\pm \mathrm{SD}$ & 1.0 & 0.9 & 0.9 & 0.6 \\
\hline Group B & Mean & 2.9 & 4.7 & 3.3 & 3.2 \\
\hline \multirow[t]{2}{*}{$(\mathrm{n}=\mathbf{5 0})$} & \pm SD & 0.7 & 1.3 & 0.5 & 0.4 \\
\hline & & 0.082 & $<0.001 *$ & $<0.001 *$ & $<0.001 *$ \\
\hline
\end{tabular}

* Significant as $\mathrm{P}$ value $<0.05$ 
Table (3) Total dose of morphine (mg) andTime to the first morphine request (h) in both studied groups.

\begin{tabular}{|c|c|c|c|c|}
\hline & & $\begin{array}{c}\text { Group A } \\
(n=\mathbf{5 0})\end{array}$ & $\begin{array}{c}\text { Group B } \\
(n=\mathbf{5 0})\end{array}$ & P value \\
\hline \multirow{4}{*}{$\begin{array}{l}\text { Total dose of } \\
\text { morphine (mg) } \\
\text { Time to the first } \\
\text { morphine request }(\mathbf{h})\end{array}$} & Mean \pm SD & $28.10 \pm 5.43$ & $12.80 \pm 1.84$ & $<0.001 *$ \\
\hline & Range & $20-40$ & $10-15$ & \\
\hline & Mean \pm SD & $2.19 \pm 0.55$ & $5.70 \pm 1.34$ & $<0.001 *$ \\
\hline & Range & $1.5-3.5$ & $3.75-7.5$ & \\
\hline
\end{tabular}

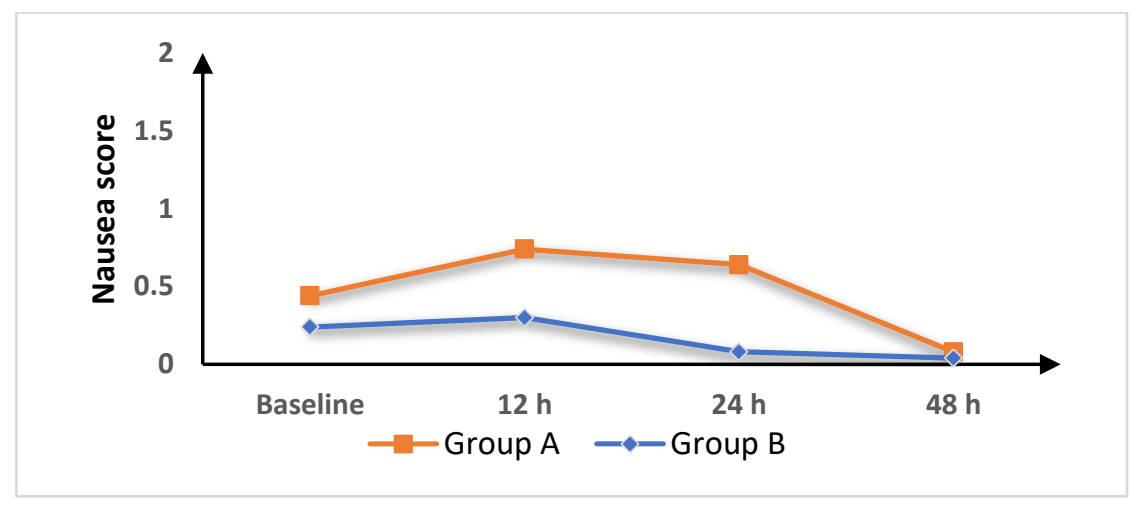

Fig. (2) Nausea score in both studied groups.

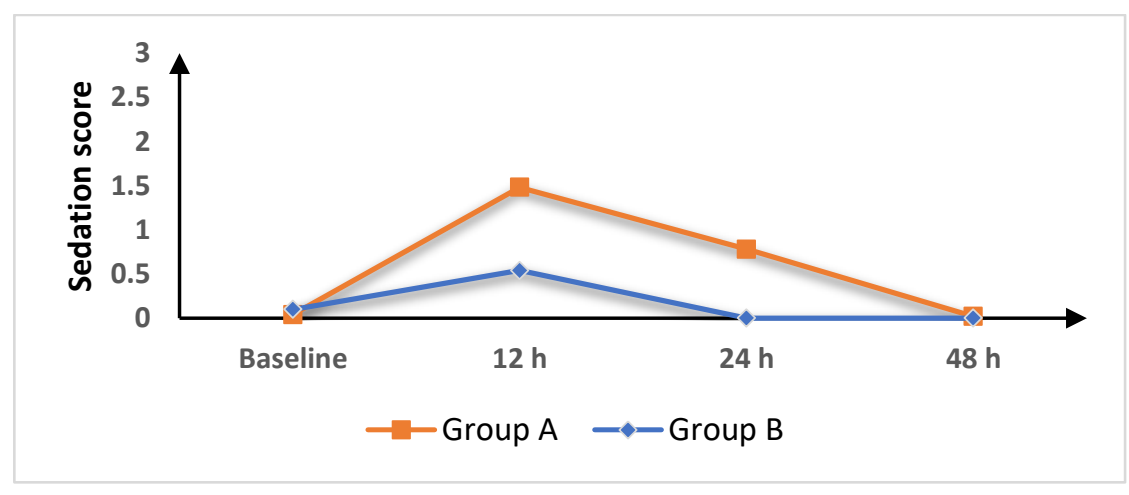

Fig. (3) Sedation score in both studied groups.

Nausea score was significantly lower in group B compared to group $\mathrm{A}$ at $12 \mathrm{~h}$ and $1 \mathrm{~d}(\mathrm{P}=0.001$ and $<0.001)$. Nausea score was insignificantly different between both groups at 0 and $2 \mathrm{~d}$ Fig. (2).

Sedation score was significantly lower in group B compared to group $\mathrm{A}$ at $12 \mathrm{~h}$ and $1 \mathrm{~d}(\mathrm{P}<0.001)$ but was insignificantly different between both groups at 0 and $2 \mathrm{~d}$ Fig. (3).

\section{Discussion}

During 12, 24, and 48 hours, VAS at rest and movement were considerably lower in group B compared to group A, although there was no difference between the two groups at baseline.

Fusco et al. [8] found that TAP block enhanced postoperative pain management and patient satisfaction after CS, which is consistent with our findings. In addition, Belavy et al. [9] found that in patients having CS, TAP block with ropivacaine 0.5 percent had a lower VAS than TAP block with saline.
According to our findings, Baaj et al. [10] found that TAP block with 0.25 percent bupivacaine had a lower VAS than TAP block with saline in individuals having $\mathrm{CS}$. In patients receiving CS, Eslamian et al. [11] found that TAP block with bupivacaine 0.25 percent had reduced VAS during rest and movement compared to no blockage. In addition, Mankikar et al [12] found that TAP block with 0.25 percent bupivacaine had a lower VAS than TAP block with saline in patients receiving CS. Our results are consistent with a meta-analysis of nine studies by Mishriky et al [13], which found that TAP block with bupivacaine 0.25 percent resulted in reduced VAS for up to 12 hours in patients having CS compared to no blocking. In addition, McDonnell et al. [7] found that TAP block with ropivacaine 0.75 percent had lower VAS at rest and with movement in patients receiving CS than TAP block with saline. Tan et al. [14], in contrast to our findings, found no significant variations in VAS between TAP block and no block. 
In our research, group B had a much longer wait before the first morphine request and a much lower overall dosage of morphine than group $\mathrm{A}$.

Fusco et al. [8], McDonnell et al. [7], and Tan et al. [14] all came to the same conclusion as us that TAP block lowered opioid usage after surgery. In addition, Eslamian et al [11] discovered that women who got TAP block used much less tramadol and waited longer for their initial analgesic request than women who did not get TAP block. Furthermore, women who underwent TAP block consumed considerably less tramadol and the initial request for analgesia was delayed compared to the control group, according to Mishriky et al. [13] and Mankikar et al. [12]. In addition, Belavy et al [9] and Baaj et al [10] found that the active group used less morphine in 24 hours than the placebo group. Pather et al. [15] examined the impact of TAP block in women having complete laparoscopic hysterectomy to those who had standard anaesthetic without TAP block, and found that TAP block reduced opioid usage relative to controls.

At 12 hours and 1 day, nausea score in group B was much lower than in group A, but at 0 and 2 days, there was no significant difference between the two groups.

In accordance with our findings, Gao et al. [16] found that postoperative problems (e.g., nausea and vomiting) were considerably reduced in TAP block compared to IV PCA. Mishriky et al. [13] found that TAP block greatly decreased nausea in individuals who did not receive intrathecal morphine, which is consistent with our findings. Baaj et al. [10], based on our findings, concluded that the bupivacaine group had less nausea and vomiting. Belavy et al. [9] also found that the TAP group experienced reduced nausea.

Tan et al. [14], in contrast to our findings, found no differences in nausea and vomiting across groups.

At 12 hours and 1 day, group $B$ had a considerably lower sedation score than group A, but at 0 and 2 days, there was no significant difference between the two groups.

According to McDonnell et al [7], TAP block lowered the frequency of sedation in the block vs control vs control vs control vs control vs control vs control vs control vs control vs control vs control vs control vs control vs control vs ( 0 percent vs 36 percent ). Tan et al. [14], in contrast to our findings, found no changes in sedation levels across groups.

\section{Conclusion}

The use of a US-guided TAP block following emergency CS reduces the need for analgesics in the first 48 hours following surgery. It lowers the overall quantity of morphine, the sedation score, and the nausea score while reducing pain intensity and prolonging the time to first rescue analgesia.

Financial support and sponsorship: Nil

Conflict of Interest: Nil

\section{References}

[1] H.Kehlet, K.Holte. Effect of postoperative analgesia on surgical outcome. $\mathrm{Br} \mathbf{J}$ Anaesth.vol.87,pp.62-72,2001.

[2] H.Kehlet, TS.Jensen, Woolf CJ. Persistent postsurgical pain: risk factors and prevention. Lancet.vol.367,pp.1618-25,2006.

[3] P.Barash, F.Cullen Bruce, K.Stoelting Robert, KM.Cahalan Michael, M.Stock, M.Christine. Clinical anesthesia. Phladelphia: Lippincott Williams and Wilkins.vol.8mpp.1-9, 2009.

[4] TO.de Brito Cançado, M.Omais, HA.Ashmawi, ML.Torres. Chronic pain after cesarean section. Influence of anesthetic/surgical technique and postoperative analgesia. Rev Bras Anestesiol.vol.62,pp.762-74,2012.

[5] C.Maggioni, D.Margola, F.Filippi. PTSD, risk factors, and expectations among women having a baby: a two-wave longitudinal study. J Psychosom Obstet Gynaecol.vol.27,pp.8190,2006.

[6] American Society of Anesthesiologist Task Force on Pain Management. Practice guidelines for acute pain management in the perioperative setting: an updated report by the American Society of Anesthesiologists Task Force on Acute Pain Management. Anesthesiology.vol.116,pp.248-73,2012.

[7] JG.McDonnell, G.Curley, J.Carney, A.Benton, J.Costello, CH.Maharaj. The analgesic efficacy of transversus abdominis plane block after cesarean delivery: a randomized controlled trial. Anesth Analg.vol.106,pp.186-91, 2008.

[8] P.Fusco, P.Scimia, G.Paladini, M.Fiorenzi, E.Petrucci, T.Pozone. Transversus abdominis plane block for analgesia after Cesarean delivery. A systematic review. Minerva Anestesiol.vol.81,pp.195-204. 2015

[9] D.Belavy, PJ.Cowlishaw, M.Howes, F.Phillips. Ultrasound-guided transversus abdominis plane block for analgesia after Caesarean delivery. BJA: British Journal of Anaesthesia.vol.103,pp.726-30,2009.

[10] JM.Baaj, RA.Alsatli, HA.Majaj, ZA.Babay, AK.Thallaj. Efficacy of ultrasound-guided transversus abdominis plane (TAP) block for postcesarean section delivery analgesia--a double-blind, placebo-controlled, randomized study. Middle East journal of anaesthesiology.vol.20,pp.821-6,2010.

[11] L.Eslamian, Z.Jalili, A.Jamal, V.Marsoosi, A.Movafegh. Transversus abdominis plane block reduces postoperative pain intensity and analgesic consumption in elective cesarean delivery under general anesthesia. Journal of Anesthesia.vol.26,pp.334-8, 2012.

[12] MG.Mankikar, SP.Sardesai, PS.Ghodki. Ultrasound-guided transversus abdominis plane block for post-operative analgesia in patients 
undergoing caesarean section. Indian J Anaesth.vol.60,pp.253-7,2016.

[13] BM.Mishriky, RB.George, AS.Habib. Transversus abdominis plane block for analgesia after Cesarean delivery: a systematic review and meta-analysis. Can J Anaesth.vol.59,pp.766-78,2012.

[14] TT.Tan, WHL.Teoh, DCM.Woo, CE.Ocampo, MK.Shah, ATH.Sia. A randomised trial of the analgesic efficacy of ultrasound-guided transversus abdominis plane block after caesarean delivery under general anaesthesia. Eur J Anaesthesiol.vol.29,pp.8894,2012.
[15] S.Pather, JA.Loadsman, PD.Gopalan, A.Rao, S.Philp, J.Carter. The role of transversus abdominis plane blocks in women undergoing total laparoscopic hysterectomy: a retrospective review. Australian New Zealand Journal of Obstetrics Gynaecology journal.vol.51,pp.5447,2011.

[16] Y.Gao, M.Guo, C.Du, H.Zhang, H.Zhang. Clinical study of ultrasound-guided transversus abdominis plane block for analgesia after cesarean section. Medicine.vol.98,pp.17582,2019 . 